\title{
Polymer electrolyte membrane fuel cell control with feed-forward and feedback strategy
}

\author{
Omar Rgab, D. L. Yu and J. B. Gomm \\ Control Systems Research Group, School of Engineering,Liverpool John Moores University, Byrom Street, Liverpool, L3 3AF, UK \\ Email:om.khazali_yo@yahoo.com
}

\begin{abstract}
Feed-forward and feedback control is developed in this work for Polymer electrolyte membrane (PEM) fuel cell stacks. The feed-forward control is achieved using different methods, including look-up table, fuzzy logic and neural network, to improve the fuel cell stack breathing control and prevent the problem of oxygen starvation. Firstly, the feed-forward controller is used to generate directly an input voltage of the compressor according to the current demand. Then, a PID controller is used in the feedback to adjust the difference between the requested and the actual oxygen ratio by compensating the feed-forward controller output. The designed system is evaluated using a nonlinear simulation of a fuel cell model documented in the literature. The proposed feed-forward with PID controller have achieved a good control performance. The simulation showed effectiveness of the control strategy.
\end{abstract}

Keywords: Fuel cell; fuel cell stack; breathing control; starvation; feed-forward; fuzzy logic; neural network.

\section{Introduction}

There are many environmental problems in the world today associated with current natural sources such as fossil fuels. Burning fossil fuels emits $\mathrm{CO}_{2}$ and destructs the ozone layer which leads to climatic change and what is known as the greenhouse effect. From this point, the world has been looking for energy sources that are clean and safe on the environment. Fuel cells are a kind of clean and safety energy source on the environment. Polymer electrolyte membrane (PEM) fuel cells emerge as one of the most clean and promising alternatives to reduce fossil fuel dependency (Pukrushpan et al., 2004a). In this paper some advanced control methods are implemented to achieve better control for the fuel cell breathing.

1.1 Fuel cell working principles: Fuel cells convert chemical energy of a hydrogen fuel (on the anode side) into electric energy with water and some heat through a chemical reaction with oxygen (on the cathode side) (Pukrushpan et al., 2004b), to satisfy different power requirements (figure 1). Generally, the reactants flow in and reaction products flow out while the electrolyte remains in the cell. Fuel cells can operate continuously as long as the necessary flows are maintained. Fuel cells differ from batteries in that they do not need recharging, they operate quietly and efficiently, and when hydrogen is used as fuel they generate only electric power and drinking water. So, they are called zero emission engines. William Grove has discovered the basic operating principle of fuel cells by reversing water in 1839 (Hoogers, 2003). In particular, proton exchange membrane FCs (PEMFCs), also known as polymer electrolyte membrane FCs, is considered to be more developed than other FC technologies, because they have high power density, solid electrolyte, operate at low temp, long cell and stack life and low corrosion (Hoogers, 2003). The PEM-FC takes its name from the special plastic membrane used as the electrolyte. This membrane electrode assembly (MEA), not thicker than a few hundred microns, is the heart of a PEM-FC and, when supplied with fuel and air, generates electric power at cell voltages around $0.7 \mathrm{~V}$ and power densities of up to about $1 \mathrm{~W} / \mathrm{cm}$ electrode area (Spiegel, 2008). 


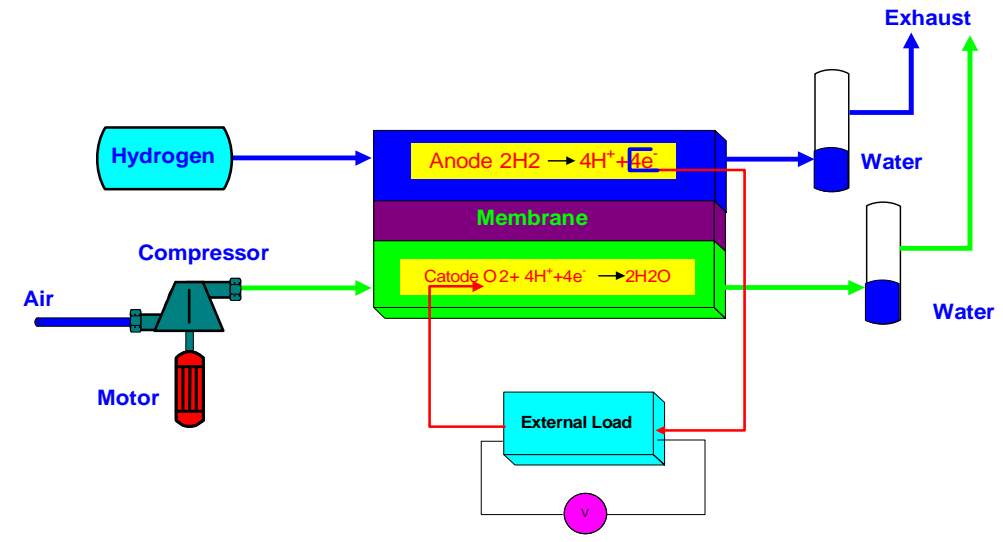

Figure1. PME- FC reaction and structure

Figure 2 shows a schematic of an MEA. The MEA is typically located between a pair of current collector plates (platinumimpregnated porous electrodes) with machined flow fields for distributing fuel and oxidant to the anode and cathode, respectively. A water jacket for cooling is often placed at the back of each reactant flow field followed by a metallic current collector plate. The cell can also contain a humidification section for the reactant gases, which are kept close to their saturation level in order to prevent dehydration of the membrane electrolyte. Many FCs are connected electrically in series to form an FC stack (FCS).

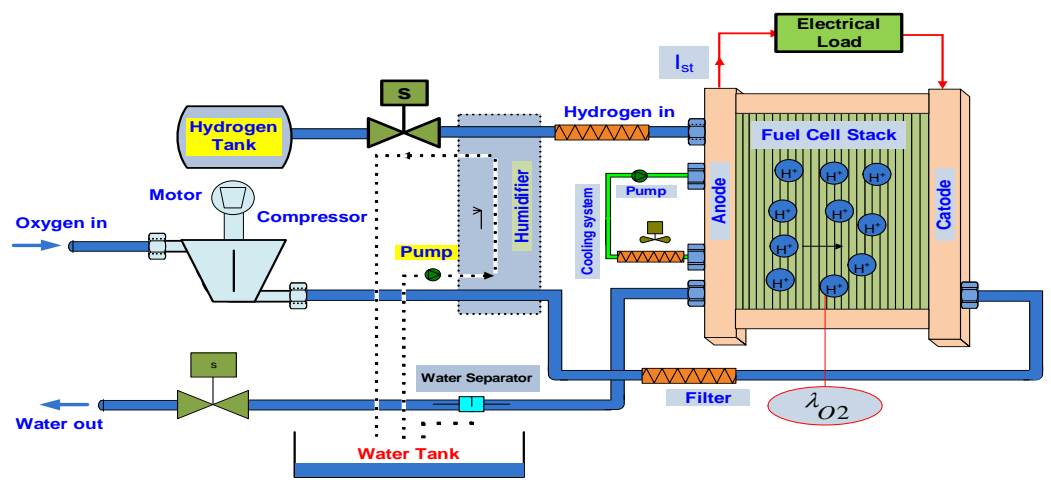

Figure 2. PEM fuel cell structure

1.2 Fuel stack model: The fuel cell stack (FCS) model simulated in this paper consists of four interacting sub-models which are the stack voltage, the anode flow, the cathode flow, and the membrane hydration models (Pukrushpan et al., 2005). The voltage model contains an equation to calculate stack voltage that based on fuel cell temperature, pressure, reactant gas partial pressures and membrane humidity. In summary, the fuel cell voltage $E$ is given by

$$
E=1.229-0.85 \times 10^{-3}\left(T_{f_{C}}-298.15\right)+4.3085 \times 10^{-5} T_{f_{C}}\left[\ln \left(p_{H 2}\right)+\frac{1}{2}\left(p_{O 2}\right)\right]
$$

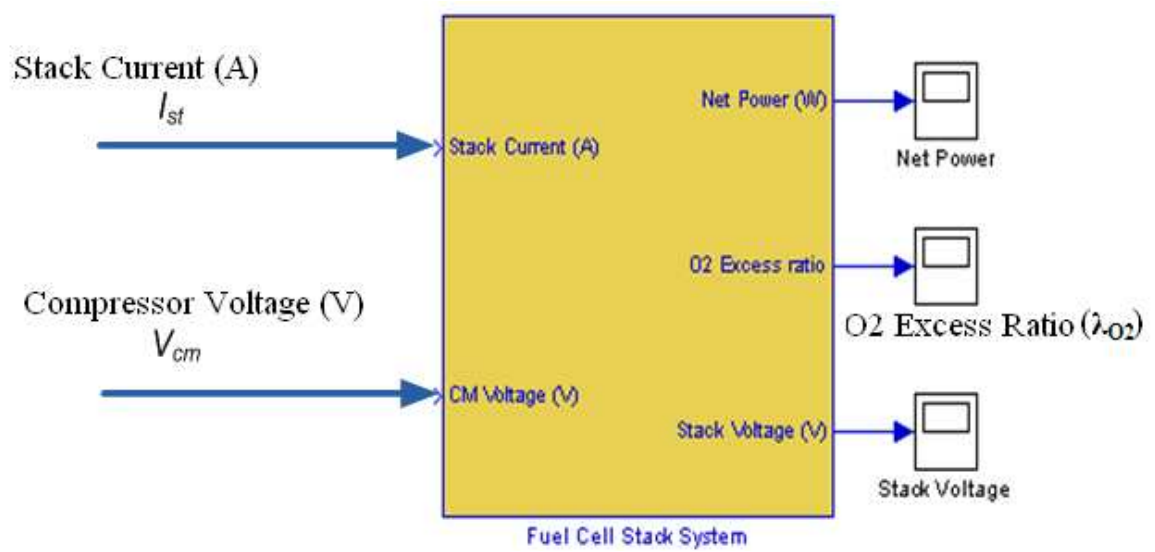

Figure 3. Simulink model of integrated PEM fuel cell 
where, $T_{f c}$ the fuel cell temperature in Kelvin, $p_{\mathrm{H} 2}$ and $p_{\mathrm{O} 2}$ are the partial pressures of hydrogen and oxygen respectively, details in Pukrushpan et al. $(2005,2004 \mathrm{c})$. In this model the stack temperature is assumed to be constant at $80^{\circ} \mathrm{C}$. The model which is used in our investigation is given in (Pukrushpan et al., 2005). The FCS Simulink model is created in Matlab 6.5.

1.3 Literature survey: The main three parameters of fuel cell stack are stack current $I_{s t}$, stack voltage $v_{s t}$ and the oxygen ratio $\lambda_{\mathrm{o} 2}$. A fuel cell (FC) stack has to be operated properly to get good power efficiency, reliability and smooth operation. The main disadvantage of the fuel cells stack system is the oxygen starvation. Since current is instantaneously drawn from the load source connected to the FC, the FC control system is required to maintain optimal temperature, membrane hydration, and partial pressure of the reactants across the membrane to avoid detrimental degradation of the FC voltage, which can reduce efficiency. Fuel cell parameters need to be controlled to avoid the oxygen starvation phenomena when the current is drawn from the fuel cell. Some researchers have presented some methods to control the breathing of fuel cell stack in order to prevent the problem of oxygen starvation, and some other problems, which are now reviewed.

Friede and Davat. (2004) proposed the governing equations of the transient behavior of a PEM fuel cell to show the influence of the operating conditions (such as temperature, pressure and gas flows have an effected on the humidity condition of the fuel cell and their choice is the greatest challenge in fuel cell operation) and the current density on internal parameters, especially the ohmic resistance to improve the control of fuel cell. The models were suitable for description of highly nonlinear behavior of the fuel cells. Vahidi et al. (2006) have used a bank of ultra capacitors supplements the fuel cell during fast current transient in order to prevent fuel cell oxygen starvation, air compressor surge and choke, and simultaneously match an arbitrary level of current demand during rapid load demands. A model-predictive controller has been designed for optimal distribution of current demand between the fuel cell and the bank of ultra capacitors, which is handled multiple constrains of the hybrid system. Pukrushpan et al. (2002) implemented a nonlinear fuel cell dynamic model for control study of fuel cell. The model has captured the transient phenomena which include the flow characteristics and inertia dynamics of the compressor, the manifold filling dynamics and consequently, the reactant partial pressures. They attempted to design an observer based feedback and feed-forward controller that manages the tradeoff between reduction of parasitic losses and fast fuel cell net power response during rapid current (load) demands. An air flow controller (Pukrushpan et al., 2004b) to protect the FC stack from oxygen starvation during step changes of current demand have been designed, the steady-state regulation of the oxygen excess ratio in the FCS cathode have been achieved by assigning an integrator to the compressor flow. Linear observablility techniques were employed to demonstrate improvements in transient oxygen regulation when the FCS voltage is included as a measurement for the feedback controller. A linear optimal control design had been used to identify the frequencies (the FCS voltage signal contains high frequency information about the FC oxygen utilization, and thus, is a natural and valuable output for feedback) at which there was a severe tradeoff between the transient system net power performance and the stack starvation control. The limitation arises when the FCS system architecture dictates that all auxiliary equipment is powered directly from the FC with no secondary power sources. An observer was designed by Arcak et al. (2004) to estimate anode hydrogen pressure with an output injection term based on stack voltage. The paper by Kunusch et al. (2009) tackles the breathing problem of subsystem of a PEM fuel cell stacks by using a second order sliding mode strategy. The proposed control strategy is based on a super twisting algorithm that robustly solves the stabilization problem avoiding chattering effects. The resulting approach exhibits good dynamic characteristics, being robust to uncertainties and disturbances, and the results were provided showed the feasibility of the approach. A method for controlling a nonlinear underactuated system (DiFiore., 2009) using augmented sliding mode control (SMC) have been investigated, where the proposed control approach involves introducing a transformation matrix mapping the systems input influence matrix to a transformed system that is square and thus invertible. The proposed approach is shown to control selectable states with proper choice of the transformation matrix yielding good control performance. From other hand, the methodology is applied to an under actuated nonlinear fuel cell system to show its viability in a real world application, then a sliding mode controller is derived for the full nonlinear system with a switching gain accounting for modeling errors and uncertainties. Simulation results indicate the viability of the proposed control law and demonstrate the robust nature of the control law in the presence of significant modeling errors while maintaining tracking stability. Finally, the augmented SMC is compared to a traditional linear control architecture illustrating the electiveness and advantages in tracking performance and control effort over traditional methods.

\section{Fuel cell control techniques}

The FC air flow needs to be controlled rapidly and capably. In order to avoid oxygen starvation and extend the life of the FC stack (Yang et al.,1998) Oxygen starvation is a complicated phenomenon that occurs when the partial pressure of oxygen falls below a critical level at any location within the meander of the air stream in the cathode (Springer et al., 2001). This phenomenon entails a rapid decrease in cell voltage, which in severe cases can causes a hot spot, or even burn-through on the surface of a membrane. Although the oxygen starvation is spatially varying, this phenomenon can be avoided by regulating the cathode excess

oxygen ratio $\lambda_{O 2}$. We thus regulate air ratio in the FCS cathode by controlling compressor motor voltage $V_{c m}$ during step changes in current drawn $I_{s t}$ from the FCS.

2.1 Feed-Forward and Feedback control methods: The fuel cell model documented in (Pukrushpan et al., 2005) will be applied to the augmented feed-back (FB) and feed-forward (FF) controllers with different design methods for the FF controller. Firstly the 
feed-forward FF controller is used to generate directly an input voltage of the compressor according to the current demand. Then, a PID controller is used in the feedback to adjust the difference between the requested and the actual oxygen ratio by compensating the feed-forward controller output. The nonlinear state equations are $\dot{x}_{N L}=f\left(x_{N L}, u, d\right), u=V_{c m}$ (Control signal), $d=I_{s t}$ (Disturbance inputs), where the control input $u$ is the compressor motor voltage $v_{c m}$, and the disturbance input $d$ is the current $I_{s t}$ drawn from the FCS. The performance variables are excess oxygen ratio $y=\lambda_{O 2}$ in the fuel cell cathode.

2.2 System configuration: System configuration includes four different control schemes for the FC stack system with FF and FB controllers as shown in figure 4, the FF neural network method will be discussed later. The disturbance (stack current $I_{s t}$ ) can be measured; FF controller that correlates the steady state value between the control input $v_{c m}$ and the disturbance $I_{s t}$ will be used in the FF path. The FF controller will implement by different methods such as a look-up table, fuzzy logic controller (5 and 9 membership function MF) and neural network. Analytical modeling or experimental testing can be used to construct the inverse of compressor and compressor motor maps to find $\dot{v}_{c m}=f_{c m}\left(I_{s t}\right)$ at desired oxygen flow $\dot{w}_{c p}$. FF controller and FB controller can be designed to achieve better transient response. In fact, a FF controller that cancels the effect of $d$ to $y$ over a wide range of frequencies is designed first. A feedback controller (PID) is designed to reduce the error in the output of oxygen ratio $\lambda_{\mathrm{O} 2}$.

2.3 Setting of PID controller parameters: PID controller equation given in (Ogata, 1997) has the following form:

$$
W c(s)=K_{p}+\frac{K_{i}}{s}+K_{d} s
$$

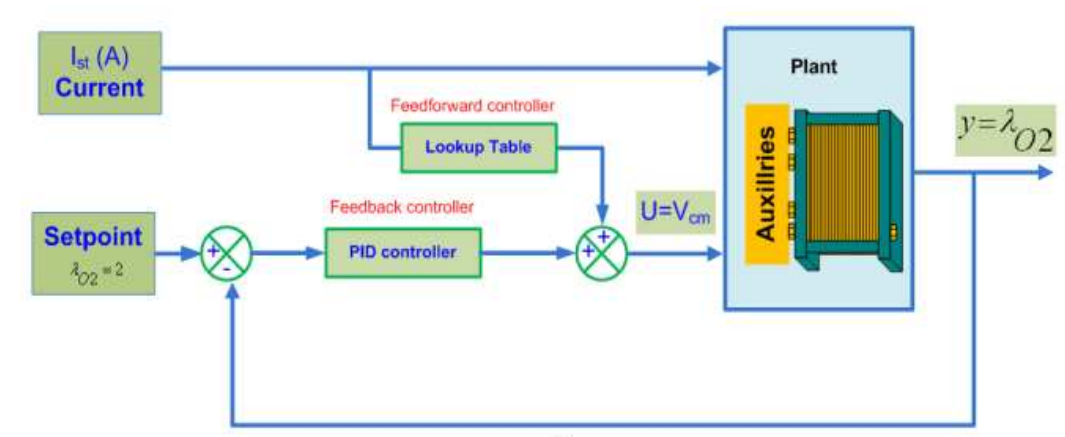

(a)

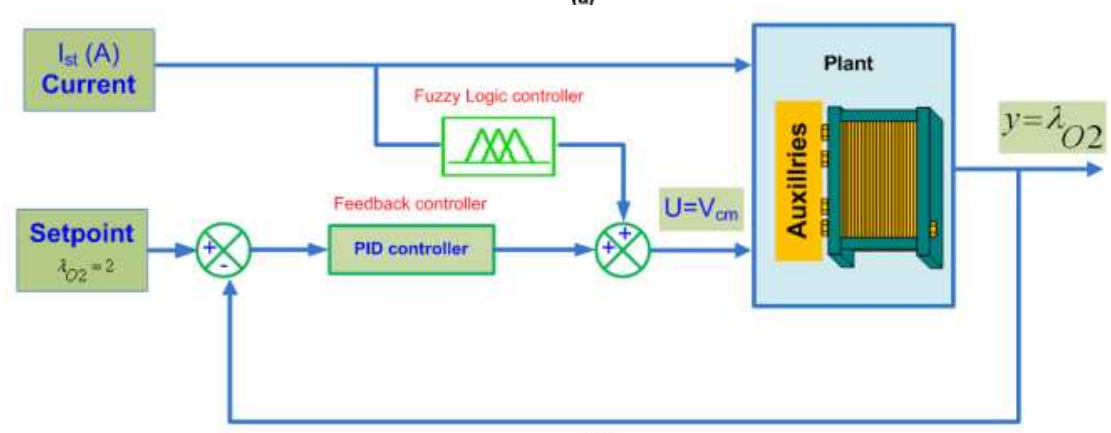

(b)

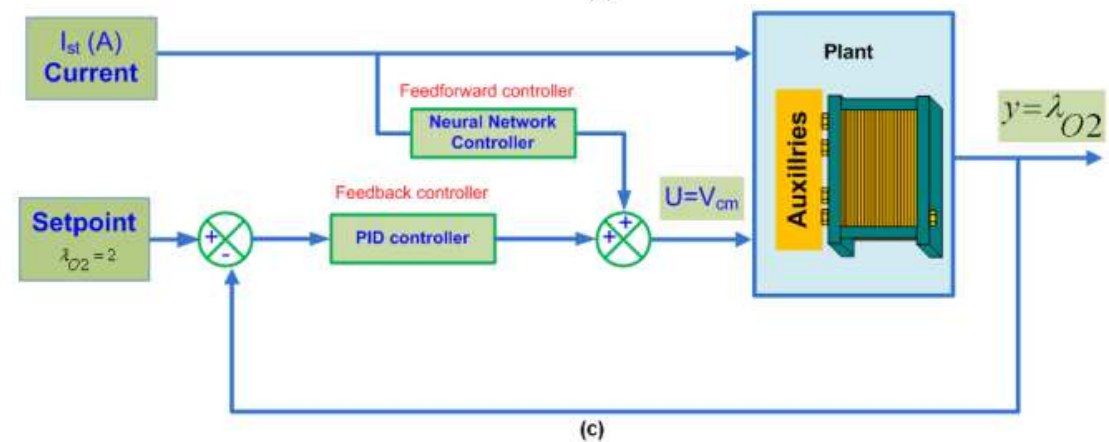

Figure 4. Fuel cell control Construction. (a) Look-up table FF controller and PID controller (b) Fuzzy logic FF (5\&9 MF.) and PID controller. (c) Neural Network controller FF and PID controller. 
where, $K_{p}, K_{i}$ and $K_{d}$ are proportional, integral and differential gains respectively. The PID is initially tuned by the Zigler and Nichols for the open loop method. The measured oxygen ratio is the Feedback signal of the system. After fine tuning, the PID controller that is used here with Feed-forward controllers for oxygen ratio regulation is

$$
W_{c}(s)=200\left(1+\frac{1}{0.6153 s}+0.05 s\right)
$$

Also, the best control results and corresponding fuel cell compressor voltages are shown in figures 9 12.

\subsection{Feed-forward controller design}

2.4.1 Look-up table feed-forward controller: The FF controller here is implemented with a lookup table configuration, see figure 4(a). The values of this table are obtained from the model by giving current values into the FCS model, and then we have used the compressor motor voltage $V_{c m}$ to regulate the oxygen excess ratio $\lambda_{O 2}=2$. Compressor voltage for each current value is illustrated in the table 1.

Table 1. Compressor voltage $\left(v_{c m}\right)$ and FCS current $\left(I_{s t}\right)$ values

\begin{tabular}{|c|c|c|}
\hline Current $\left(I_{s t}\right)$ & Compressor voltage $\left(v_{c m}\right)$ & $\mathrm{K}=$ voltage /current \\
\hline 100 & 100 & 1 \\
\hline 110 & 105.96 & 0.9633 \\
\hline 120 & 112 & 0.9333 \\
\hline 130 & 120.42 & 0.9263 \\
\hline 140 & 127 & 0.9071 \\
\hline 150 & 134.9 & 0.8993 \\
\hline 160 & 142 & 0.8875 \\
\hline 170 & 149 & 0.8764 \\
\hline 180 & 156 & 0.9166 \\
\hline 190 & 163 & 0.8579 \\
\hline 200 & 170 & 0.8500 \\
\hline 210 & 176.5 & 0.8404 \\
\hline 220 & 183.2 & 0.8327 \\
\hline 230 & 189.595 & 0.8240 \\
\hline 240 & 195.88 & 0.8162 \\
\hline 250 & 202 & 0.8080 \\
\hline 260 & 208 & 0.8000 \\
\hline 270 & 214.2 & 0.7933 \\
\hline 280 & 220.49 & 0.7875 \\
\hline 290 & 227 & 0.7828 \\
\hline 300 & 233.57 & 0.7786 \\
\hline
\end{tabular}

2.4.2 Feed-forward fuzzy logic controller: The fuzzy logic control technique can be applied to control of the fuel cell, since the fuzzy logic is relatively simple and is based on definitions of formal facts and the relationships among them. In figure 4(b), the fuzzy controller consists of the following main elements (Tsoukalas and Uhrig, 2000; Popovic and Bhatkar, 1997):

- $\quad$ A set of control rules: IF (condition) Then (control actions), where "condition" defines the state of the process, for which the control adjustment specified in the control action should be executed. These rules are derived from the knowledge of experts with substantial experience in the system.

- $\quad$ Membership functions MF: which are a simple yet versatile mathematical tool for indicating flexible memberships to a set, see figures $(5 \mathrm{a} \& 5 \mathrm{~b})$. Fuzzy numbers are fuzzy subsets of the real line. They have a peak or plateau with membership grade 1, over which the members of the universe are completely in the set. The membership function is increasing towards the peak and decreasing away from it. Fuzzy membership functions are used in fuzzy control applications. A typical case is the triangular fuzzy membership function and is used in this studies (fig.5a \&5b).

- Fuzzification interface (the input of fuzzy controller) is used to transform a crisp set into a fuzzy set or which transforms the control variables into fuzzy sets manipulated by a collection of fuzzy rules, assembled in what is known as the fuzzy inference engine (Tsoukalas and Uhrig, 2000). The fuzzy control has the following characteristics. 


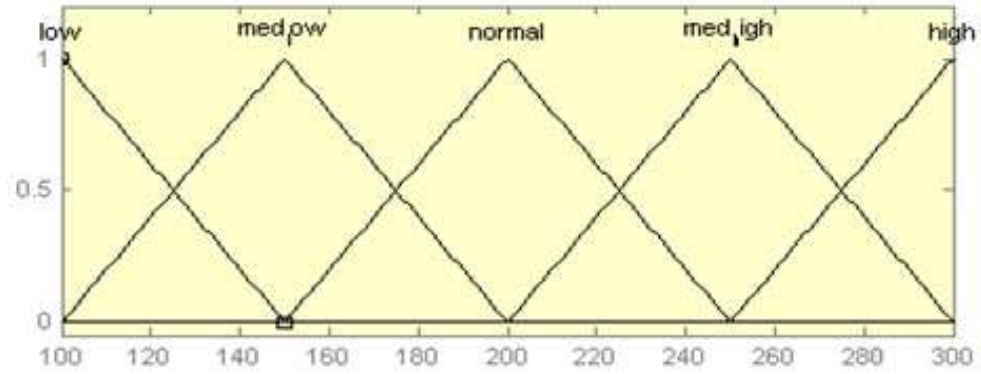

Figure 5(a). Input variable "stack current" membership"s function

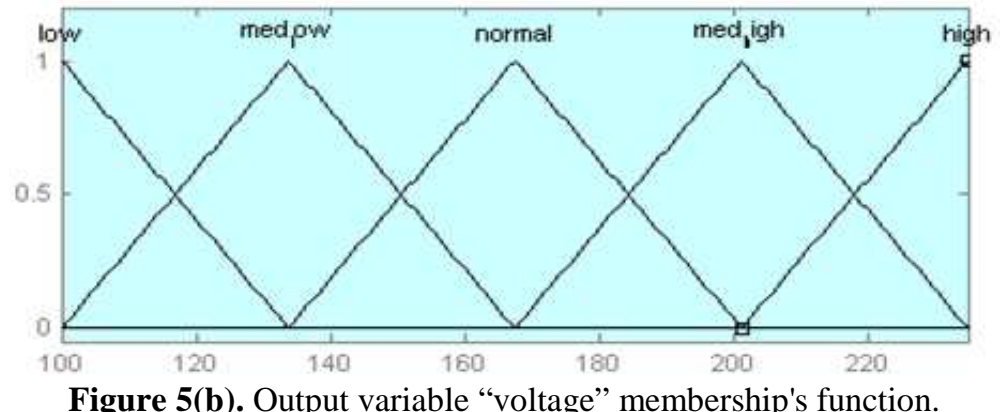

2.4.2.1 Five membership fuzzy logic controller: Fuzzy controller is designed as case of process, where the inputs of fuzzy controller is the fuel cell stack current "100 300A" (the current demand), and its output $\Delta$ output (the change of compressor voltage 100 235 volt, see table 1). The membership function for the input and output fuzzy logic controller is divided to low, med low, normal, med high, high. From the table 1, we can write the rules, which should be applied for the mentioned above membership functions as the following way in table 2 :

Table 2. Fuzzy logic controller rules

\begin{tabular}{|l|l|l|l|}
\hline $\mathrm{R}_{1^{-}}$ & If current is low & ien $\Delta$ voltage is low & Else \\
$\mathrm{R}_{2^{-}}$ & If current is med low & 1en $\Delta$ voltage is med low & Else \\
$\mathrm{R}_{3^{-}}$ & If current is normal & 1en $\Delta$ voltage is normal & Else \\
$\mathrm{R}_{4^{-}}$ & If current is med high & 1en $\Delta$ voltage is med high & Else \\
$\mathrm{R}_{5^{-}}$ & If current is high & len $\Delta$ voltage is high & Else \\
\hline
\end{tabular}

2.4.2.2 Nine membership fuzzy logic controller: In the same above principle in item 2.4.2.1, the fuzzy logic controller with $9 \mathrm{MF}$ is designed but the number MF and fuzzy logic will be different (9 MF and 9 rules). From figure 10, we can see clearly that there is a small over shoot at the first step when we used $5 \mathrm{MF}$ fuzzy logic controller with delay time at the sixth. However 9 MF fuzzy logic controller recovered this problem as shown in figure 11.

2.4.3 Neural network feed-forward controller: Neural network technique can be applied in the controlling of the fuel cell stack system, see figure 4(c), since the control process is a multi-variable with non-linear behavior. The neural network here will be used to design the FF controller because the neural net work can estimate the compressor voltage value which corresponding for any current demand value. The details of neural network structure are in (Tsoukalas and Uhrig, 2000; Haykin, 1999). The data, which is given in table 1, will be used to train the neural network. The neural network structure is illustrated in figure 6 . The values of the fuel cell current $\left(I_{s t}\right)$ will be as an input to neural network model. In this case the input layer of neural network is one, see figure 6 , and other parameters (voltage and $\mathrm{k}$ value) as an output signals. In this case the output layer has two outputs $y_{1}, y_{2}$. The number of hidden layer nodes is chosen to be 9 bases on the test performed in this work. According to the test the network with 9 hidden layer nodes is a more appropriate one and gives very small absolute error between desired and calculated output (about $1.463 \times 10^{-9}$ ). The input and hidden layers is connecting by weights $w l_{i}$ and its number is 9 as matrix $(9 \times 1)$. The hidden layer and outputs layer are connected by weights $\mathrm{w} 2_{\mathrm{ij}}$ its number is 18 (a matrix $2 \times 9$ ). Bias layers $b l_{j}$ has value equal to 1 (Tsoukalas and Uhrig, 2000) are connected with the hidden layers by weights $w b_{1 j}$ as matrix $9 \times 1$. Also bias layers $b_{2 i}$ should be introduced, its value is equal to 1 and connected to the output layers by weights $w b_{1 i}$ as matrix $2 \times 1$. In order to demonstrate supervised learning, the neural network includes the desired output vector $O$ with the components $O_{1}, O_{2}$ the computed output vector $Y$ with the component $y_{1}, y_{2}$, comparator, and weight-adjusting algorithm, this arrangement is shown in figure 6. In order to start the process, all weights in the neural network (figure 6) are randomly adjusted to small random value (-1 1) (Haykin., 1999). When the current values $I_{s t}$ is 
applied to the neural net work, it produces an output vector $Y$, which is compared with the vector $O$ by the comparator to produce the error vector $e$ calculated by equation (4). The error is applied to weight-adjusting algorithm to adjust the weights.

$$
e=O-Y
$$

The last process is repeated over and over until the error is reduced to some specified value or an irreducible small quantity. At that point the output vector $Y$ and the desired output vector $O$ are substantially equivalent, and the neural network is said to have been trained to map input vector $I_{s t}$ into the desired output vector $O$ The training procedure for the mentioned neural network (figure. 6) with the data of the fuel cell (table 1) as follows: the calculation is done by Matlap version 2009a according to the following equation:

At first, the random values between $(-1,1)$ for the weights $\left(w 1_{j}, w b 1_{j}, w 2_{i j}, w b 2_{i}\right)$ to calculate the output $Y_{j}$ of neural net work (figure 6) are introduced.

$$
\begin{aligned}
& V_{i}=w 1_{i} \cdot I_{s t}+b 1_{i} \times w b 1_{i}, i=1, \ldots \ldots ., 9 \\
& u_{i}=\operatorname{Tanh}\left(V_{i}\right), i=1, \ldots \ldots ., 9
\end{aligned}
$$

where $V i$ is the summation part and $u_{j}$ is the activation part of hidden layers (Tsoukalas and Uhrig, 2000), where, $w 1 j$, the weights which connect between the input layers and the hidden layers. Its numbers are 9 as matrix $(9 \times 1), b_{1 j}$ bias layers. Its number is 9 and has values equal to $1, w b_{j}$, the weights which connect between the bias layers and hidden layers their numbers are 9 as matrix $(9 \times 1)$. The output $y_{i}$ is calculated from the following equation (Tsoukalas and Uhrig, 2000).

$$
y_{j}=\sum_{i=1}^{9}\left(u_{i} \times w_{i j}\right)+b 2_{i} \times w b 2_{i}
$$

where; $w_{j i}$ is the weights which connect between the hidden layer and the output layer (Figure 6), and their numbers are 18 as matrix ( $2 \times 9), b 2_{i}$ Output bias layers, their numbers are 2 and have values equal to $1, w b 2_{i}$, the weights, which connect between the output bias layers $b 2_{i}$ and the output layers $y_{j}$ (figure 6) their numbers are 2 as matrix $(2 \times 1)$. The error " $e$ " is calculated from the following equation:

$$
e_{j}=O_{j}-y_{j}
$$

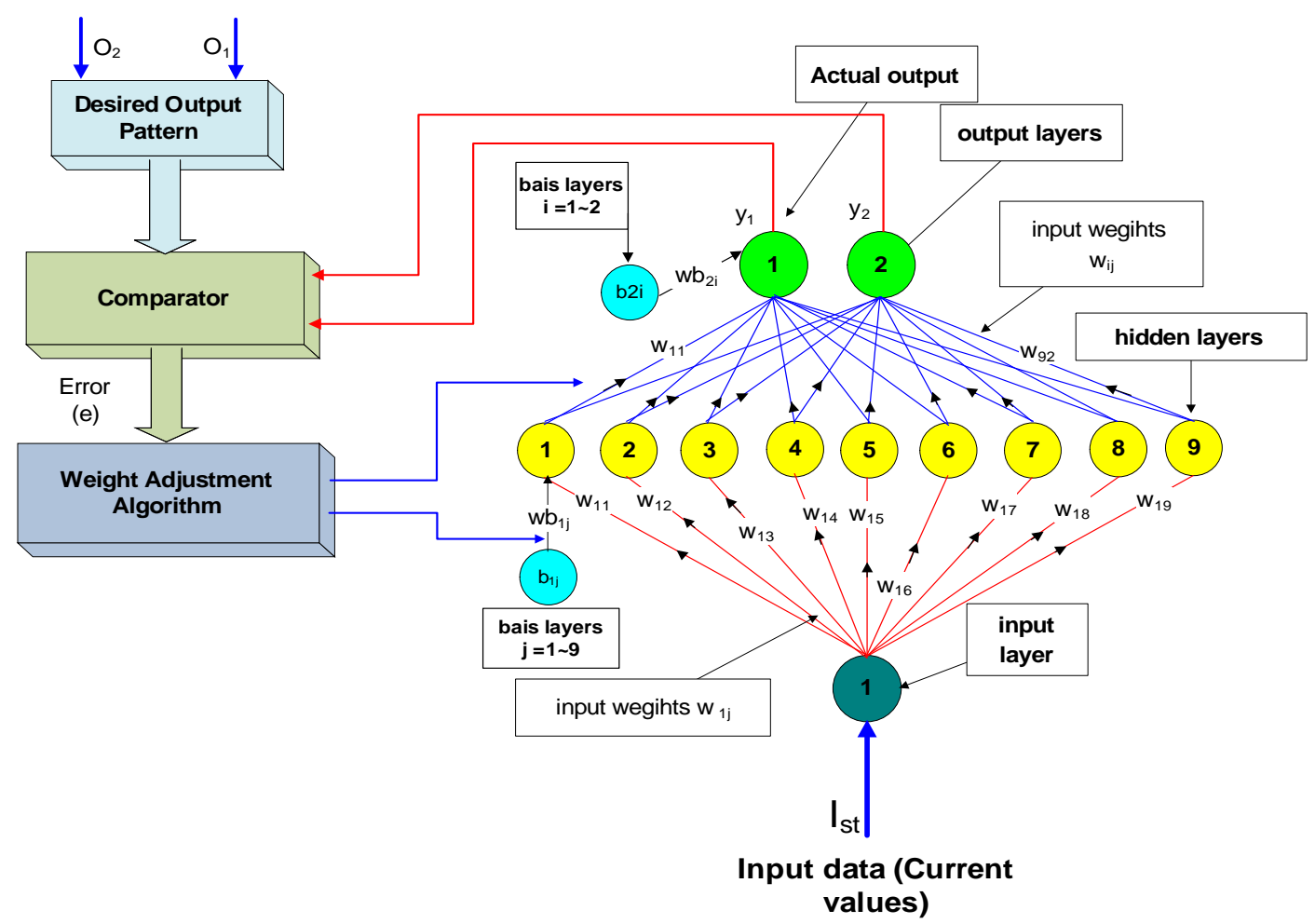

Figure 6. Neural network Model with Supervised Learning. 
where $O_{j}$, the output given in operational data or desired output, $Y_{j}$, the output computed by the neural network, $j=(1 \sim 2)$. The weights of the network are adjusted by applying back-propagation function to minimize the error. $\Delta w_{i j}$ (The change in the weights $w_{i j}$ ) is calculated from liner back-propagation function given in (Tsoukalas and Uhrig, 2000). The steps mentioned above are done for each current value (about 21 values), and repeated with changing the number of hidden layers and the type of activation function until we obtain the beast case (errors go to zero). The weights values which are corresponding to the zero error are recorded, and then the training for the neural network of figure 6 is completed and the output of $\mathrm{NN}$ can be calculated from equation (8), the Matlap version 2009a is used in our calculation.

$$
y=w \times u+b 2 \times w b 2
$$

where, $w$ is a matrix $(2 \times 9), u$ is $(9 \times 1)$ and $b 2 \times w b 2$ is matrix $(2 \times 1)$

2.5 Simulations and Evaluation: Quality of controlling is defined by building up the output response of the fuel cell stack, and determines the values of Mean Absolute Error (MAE), where

$$
M A E=\frac{1}{N} \sum_{\mathrm{k}=1}^{\mathrm{N}}|2-y(k)|=\frac{1}{N} \sum_{\mathrm{k}=1}^{\mathrm{N}}|e(k)| .
$$

The current demand changing depends on the type of the external electrical load. Usually the current in low demand is 100 Ampere. The maximum current demand is about 300 Ampere. In our simulations, as shown in figure 7, a current demand changing gradually from 100 to 300 each 4 second. This almost covers the whole fuel cell stack operating condition.

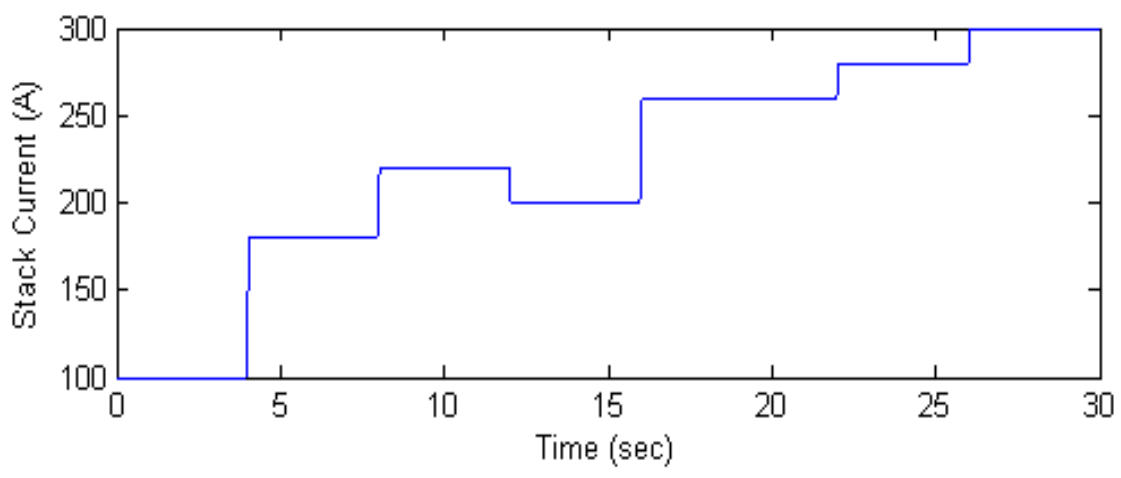

Figure 7. Current demand changing during control.

The compressor voltage is to be controlled between the 100 and 235 voltage and the oxygen ratio is to be controlled between the $-0.8 \%$ and $+0.2 \%$ bounds of ideal value $\lambda_{O 2}=2$, i.e. $99.2 \% \times 2 \leq O_{2} \leq 100.2 \% \times 2$. The output response of the FCS by using the suggested control methods is compared with the output response of the traditional Proportional Feed-forward controller (PFF with $k=164 / 191)$ as shown in figures $8,9,10,11$ and 12 .

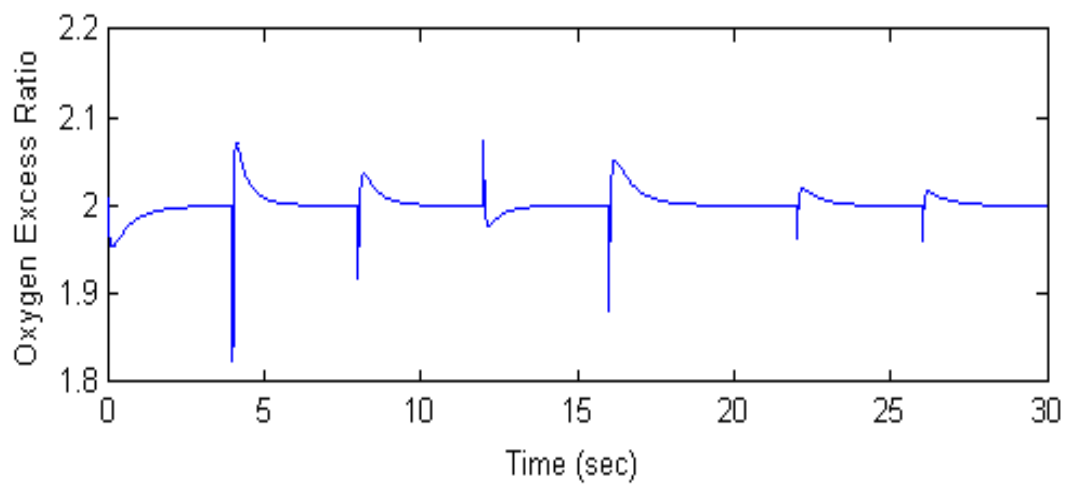

Figure 8. Output Response of FCS with proportional and PID controllers at different stack current 


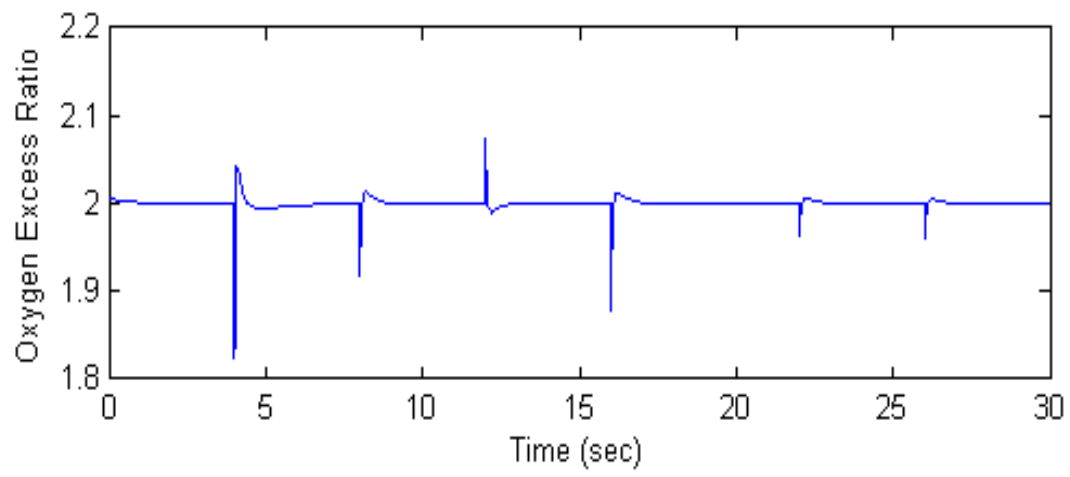

Figure 9. Output response of fuel cell control loop by using lookup table Feed-Forward Controller

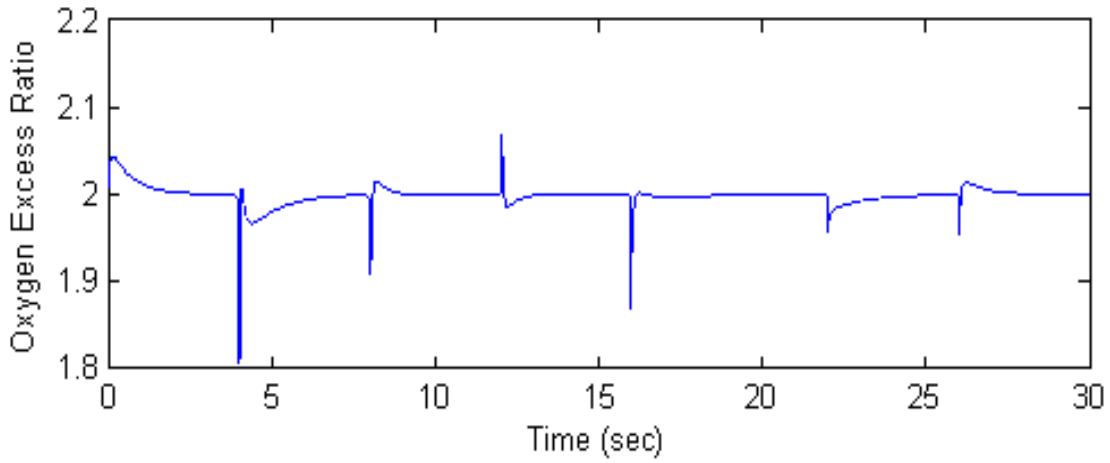

Figure 10. Output response of fuel cell control loop by using 5 MF. FL Feed-Forward controller

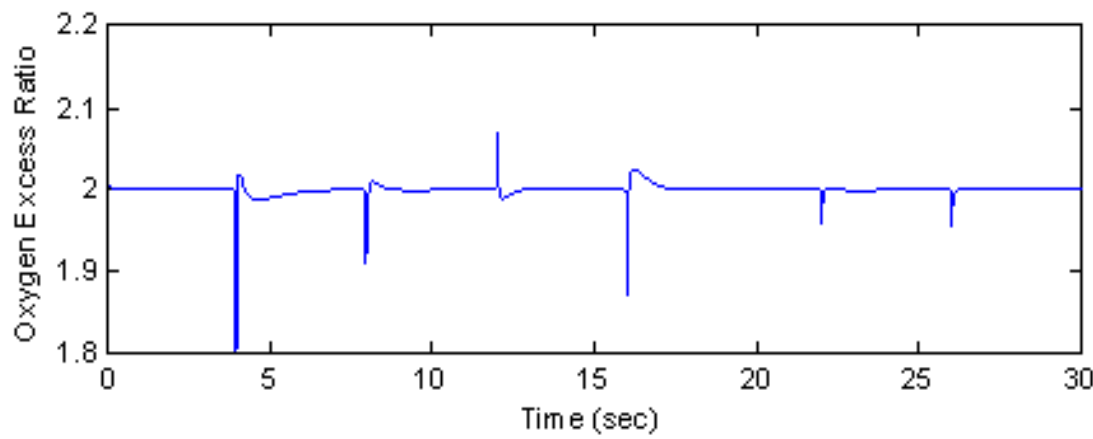

Figure 11. Output response fuel cell stack automatic control loop by using 9 MF. Fuzzy Logic controller

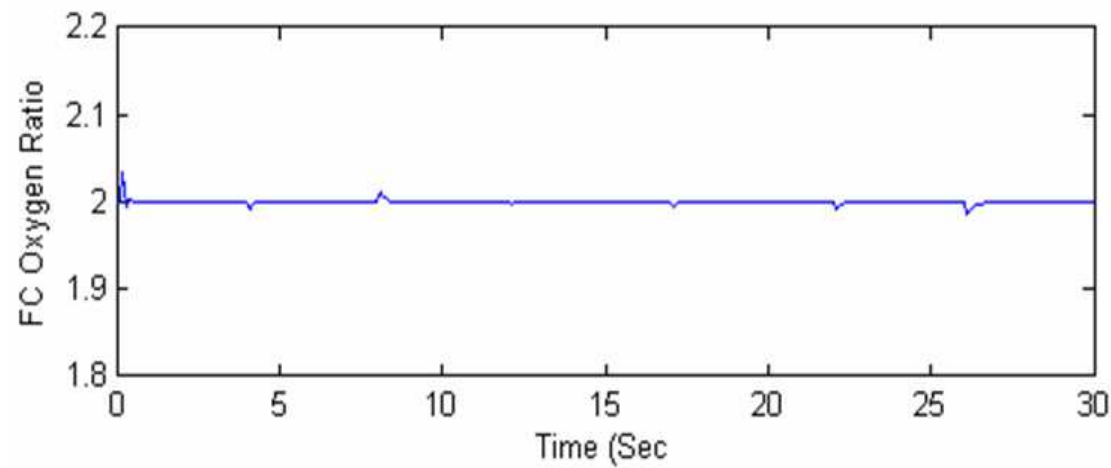

Figure 12. Output Response of FCS with neural network and PID controllers at different stack current

So, from the figures we can see clearly that the Feed-forward neural network (FF-NN) has better performance than other controllers. The second performance is that using look-up table. Then, fuzzy logic with $9 \mathrm{MF}$ followed by $5 \mathrm{MF}$ and the last one is the PFF. However, the figure 8 shows a delay time at the first step about $4 \mathrm{sec}$ in the output response of oxygen ratio when the Proportional Feed-forward Controller is used, in the second step there is about $2.5 \%$ overshoot as the same as $5^{\text {th }}$ step. In the same time the FF-NN, look-up table, $9 \& 5$ MF controllers reduced this delay time and the overshoot as shown in figures $9,10,11$ and 12 . 
Table 3, Evaluated Results

\begin{tabular}{|l|c|}
\hline Controller's type & Mean Absolute Error(MAE) \\
\hline PID FB Controller P+ FF Controller & $1.9468 \times 10^{-4}$ \\
\hline PIDFB Controller+ Lookup table FF & $4.8659 \times 10^{-5}$ \\
\hline PIDFB Controller+5 MF. FL controller & $1.1893 \times 10^{-4}$ \\
\hline PIDFB Controller +9MF. FL Controller & $6.430 \times 10^{-5}$ \\
\hline PIDFB Controller +NNFF Controller & $6.2163 \times 10^{-6}$ \\
\hline
\end{tabular}

The results of simulation and evaluation by MAE are summarized in Table 3.

\section{Conclusion}

Based on the results summarized in Table 3, it is found that:

- Neural Network FF controller + FB controller, they give better performance than other methods of control.

- The difference between five and nine memberships function is about 0.00005463 of MAE and 9 MF is better than 5 MF, but of them are better than proportional Feed-forward controller.

- The mean absolute error of Look-up table FF controller + FB controller is $4.8659 \times 10^{-5}$. So, we can say Lookup table is better performed than Proportional Feed-forward controller.

- The neural network FF controller has better performance than other controllers because the neural network can estimate the corresponding compressor voltage for each current demand, it's mean absolute error is $=6.2163 \times 10^{-6}$.

$\begin{array}{ll}\text { Abbreviations } & \\ \text { PEM } & \begin{array}{l}\text { Polymer electrolyte membrane } \\ \text { membrane electrode assembly }\end{array} \\ \text { MEA } & \text { Feed-forward } \\ \text { FF } & \text { Proportional integral differential controller } \\ \text { PID } & \text { Feed Back } \\ \text { FB } & \text { Fuel cell } \\ \text { FC } & \text { Polymer electrolyte membrane fuel cell } \\ \text { PEMFC } & \text { Fuel cell stack } \\ \text { FCS } & \text { Member ship function } \\ \text { MF } & \text { Neural net work } \\ \text { NN } & \text { Member ship fuzzy logic controller } \\ \text { MF FL } & \text { Feed forward neural net work } \\ \text { FF-NN } & \text { Proportional feed forward } \\ \text { PFF } & \end{array}$

\section{References}

Arcak, M., Gorgun, H., Pedersen, L. M., Varigondab, S., 2004. A nonlinear observer design for fuel cell hydrogen estimation. IEEE Transactions on Control Systems Technology, Vol. 12, No. 1, pp. 101-110.

DiFiore, D. C., 2009. Sliding mode control applied to an underactuated fuel cell system, Rochester Institute of Technology Rochester, New York.

Friede, W., Davat, B., 2004. Mathematical model and characterization of the transient behavior of a PEM fuel cell. IEEE Transactions on Power Electronics, Vol. 19, No. 5, pp 1234-1241.

Hoogers, G., 2003. Fuel Cell Technology Hand Book, Trier University of Applied Sciences, CRC Press LLC.

Haykin Simon., 1999. Neural Network a Comprehensive Foundation., Second ed. Prentice-Hall. Inc. New Jersey.

Kunusch, C., Puleston, P. F., Mayosky, M. A., Riera, J., 2009. Sliding mode strategy for PEM fuel cells stacks breathing control using a super-twisting algorithm, IEEE Transactions on Control Systems Technology, Vol. 17, No. 1, pp. 167-173.

Vahidi, A., Stefanopoulou, A., Peng, H., 2006. Current management in a hybrid fuel cell power system: a model predictive control approch, IEEE Transactions on Control Systems Technology, Vol. 14, No. 6, pp. 1047-1057.

Ogata, K., 1997. Modern Control Engineering. Third ed. New Jersey. 
Pukrushpan, J. T., Stefanopoulou, A., Peng, H., 2004. Control of fuel cell power systems. Springer, Vol. 13, No. 1, pp 3-14.

Pukrushpan, J. T., Stefanopoulou, A., Peng, H., 2002. Modeling and control for PEM fuel cell stack system. Proceedings of the American Control Conference Anchorage, AK, Vol. 5, pp. 3117-3122.

Pukrushpan, J. T., Stefanopoulou, A., Peng, H., 2004a. Control of fuel cell breathing. IEEE Control Systems Magazine. Vol. 24, No. 2, pp. 30-46.

Pukrushpan, J. T., Stefanopoulou, A., Peng, H., 2004b. Control of fuel cell power systems principles: modeling, analysis and feedback design, Springer-Verlag London Limited.

Pukrushpan, J. T., Stefanopoulou, A., Peng, H., 2005. Control of natural gas catalytic partial oxidation for hydrogen generation in fuel cell applications, IEEE Transactions on Control Systems Technology, Vol. 13, No. 1, pp 3-14.

Popovic, D., Bhatkar V. P., 1997. Distributed computer control for industrial automation. New York.

Spiegel, C., 2008. PEM Fuel Cell modeling and simulation using Matlab, Academic Press is an imprint of Elsevier.

Springer, T.E., R. Rockward, T.A. Zawodzinski, and S. Gottesfeld, 2001. Model for polymer electrolyte fuel cell operation on reformate feed, J. Electrochem. Soc., Vol. 148, No.1, PP. A11-A23.

Yang, W. C., Bates, B., Fletcher, N., Pow, R., Control challenges and methodologies in fuel cell vehicle development, SAE international, Paper No. 98C054.

Tsoukalas, H. L., Uhrig, E. R., 2000. Fuzzy and neural approaches in engineering. John Wiley- Interscience Publication, New York.

\section{Biographical notes}

Engineer Omar Ragb received B.Eng. degree in electrical engineering and automatic control from Bright Star University of Technology, Libya, in 1994, and M.sc. in automatic control engineering from Tabbin Institute for Metallurgical Studies, Egypt, in 2006. He was an Automatic Control and Instruments Engineer at Derna Cement Plant, Libya (1996-1998), he was as an Automatic Control and Instruments Engineer at Petroleum Golf Company, Libya (1998-1999), he was Electrical engineer at Al-Gabble Alakther dairy factory, Libya (2001), he was a Demonstrator in electrical engineering department at Omar Al-Mukhtar University, Libya ( 2002 -2003), he was a Lecturer assistant in Electrical Engineering Department at Omar Al-Mukhtar University Libya (2006- 2008), He is now a PhD student in school of engineering at Liverpool John Moores University (LJMU), UK, and His research interests include control systems research, Using a programmable logic controller in automatic control system, Cement rotary kiln control research. His current research interests include advanced control systems for a fuel cell systems, adaptive neural networks and their control applications, model predictive control for fuel cell stack, in these areas he has published 2 conference papers and preparing 2 journals.

Professor Dingli Yu received B.Eng from Harbin Civil Engineering College, China in 1982, M.Sc from Jilin University of Technology (JUT), China in 1986, and the PhD from Coventry University, U.K. in 1995, all in Control Engineering. Dr. Yu was a lecturer at JUT from 1986 to 1990, a visiting researcher at University of Salford, U.K. in 1991, a post-doctoral research fellow at Liverpool John Moores University (LJMU) from 1995 to 1998. He joined LJMU Engineering School in 1998 as a Senior Lecturer and was promoted to a Reader in 2003, then to Professor of Control Systems in 2006. He is the associate editor of two journals, International Journal of Modelling Identification and Control and International Journal of Information \& Systems Sciences. He organized two special issues in 2006, "Fault Detection, Diagnosis and Fault Tolerant Control for Dynamic Systems" and "Intelligent Monitoring and Control for Industrial systems". He serves as a member of the IFAC SAVEPROCESS Committee, and has been IPC member for many international conferences. He is a fellow of IET and Senior Member of IEEE. He leads the Control Systems Research group at LJMU. His current research interests include fault detection and fault tolerant control of bilinear and nonlinear systems, adaptive neural networks and their control applications, model predictive control for chemical processes and automotive engines and real-time evaluations, in these areas he has published more than 160 journal and conference papers.

Dr. J. Barry Gomm received the B. Eng. first class degree in electrical and electronic engineering in 1987 and the Ph. D. degree in process fault detection in 1991 from Liverpool John Moores University (LJMU), UK. He joined the academic staff at LJMU in 1991 and is a reader in intelligent control systems. His research interests include neural networks for modeling, control and fault diagnosis of non-linear processes, intelligent methods for control, system identification, adaptive systems, chemical process, and automotive applications.

Received June 2010

Accepted November 2010

Final acceptance in revised form December 2010 